\title{
Empirical Study of Tracking down the Upper-Reaches \&Lower-Reaches Suspects in the Drug-Related Cases in Shaanxi Province of the People's Republic of China \\ Min $\mathrm{CHEN}^{1}$ and Dao-Yong TU²
}

1. School of Humanities \& Foreign Languages, Xi'an University of Technology, Xi'an, (710054); Law School, Wuhan University, Wuhan (430072)

2. Shaanxi Provincial Higher People's Court, Xi'an (710061) marcy666@163.com, 1725743175@qq.com.

Keywords: Drug-related cases; Upper-reaches and lower-reaches suspects; Investigation

\begin{abstract}
Drug-related crimes, which threaten the existence and development of human being, have become a major social problem in China in recent years. This study focuses on the traits of drug-related crimes, discusses on how to effectively track down the upper-reaches and lower-reaches suspects in the drug-related cases by the public security organs, and puts forward their own solutions to the problems in such tracking-down processes.
\end{abstract}

\section{Introduction}

It is generally accepted that the drug-related crimes are an international phenomenon. In fact, this is also a common problem that the Chinese authorities are facing in its social transition. The drug-related crimes do not only hinder the economic development in China, but also destroyed many happy families, and caused more and more social instabilities. In judicial practice, the investigation organ fails to find out the upper-reaches and lower-reaches suspects in the drug-related crimes, which cannot effectively strike down such crimes in China. In this context, the first author made use of an opportunity of working in a provincial higher people's court while retaining the position in her formal affiliation during 2015-2016, who cooperated with the second author in analyzing 56 sample cases that the court reviewed in 2015, apart from the interviews with the police officers responsible for detecting drug-related crimes. This paper consists of five parts, in which it first introduces the research background, discusses the traits of the drug-related cases in Shaanxi Province, then analyzes the possible reasons that the police fail to track down the upper-reaches and lower-reaches suspects in the drug-related cases, and proposes some measures or thoughts for further improvement, hoping to effectively strike against such crimes in China.

\section{Overview of the Drug-related Cases in Shaanxi Province and the Traits of Tracking Down the Upper-reaches and Lower-reaches Suspects by the Investigation Organs}

Overall, the sources of drug-related cases in Shaanxi are mainly concentrated in such provinces as Sichuan, Guangdong and Yunnan, which has basically formed a pattern of "drug trafficking from the south to the north". The suspects detected were often the intermediate of the crime chains, as the investigators kept their eyes fixed on the drugs. Therefore, it is not a surprise for us to see that they often seized the intermediate in drugs transaction. Moreover, lots of the lower-reaches suspects tracked down by the investigation organ are addicts in Shaanxi Province. Accordingly, Shaanxi was changed from a province of "drug-transit province" into the province of having the "drug transit", "drug consumption", and "drug consumption distribution". 


\section{Analysis of the Reasons for the Failure to Track Down the Upper-Reaches and Lower-Reaches Suspects in Drug-Related Cases in Shaanxi Province}

\section{Single Clues and Various Drawbacks in Detecting the Drug-related Crimes}

1. The statements of the arrestees lacked the nature of "directivity" and "accessibility". The clues of tracking down the upper-reaches and lower-reaches suspects are from statements of the arrestees, but in practice, such statements would have a double-edged effect which may cause certain problems in the investigation. What the information provided by the upper-reaches and lower-reaches criminal suspects had no or few or unclear direction that can be used by the investigation organ in digging out more drug-related suspects. Thus the accessibility and traceability problems are the main obstacles of tracking down the upper-reaches and lower-reaches suspects in drug-related cases.

2. The sources of clues in the mode of proactive investigation are over the barrel. Comparing with the ordinary crimes, there are no or few victims in the drug-related cases; and accordingly, the clues rely heavily on the statements of addicts in the mode of proactive investigation. However, such clues will be delayed after various levels of information dissemination and the investigation organ may lose the best timing in the detection of cases.

\section{Inadequate Application of Different Means of Investigation Becomes an Obstacle for Tracking down the Crimes}

1. Technical investigation. Although the Criminal Procedure Law of the People's Republic of China (Hereinafter referred to as the "CPL") (2012) acknowledged the legal status of technical investigation, but the Ministry of Public Security (Hereinafter referred to as the "MPS") has not provided concrete measures and supporting systems for the implementation. This is why the technical investigation hits against a wall everywhere in judicial practice as there are strict requirements in the application of technical investigation which may influence the timing of tracking down the crimes. In another word, it is another obstacle on how to transform the information collected through technical investigation into lawful evidences in practice.

2. Secret investigation. In practice, it is very likely for the secret investigation to easily touch the "high-voltage wire" of joint crimes when tracking down the upper-reaches and lower-reaches suspects, because of the difficulties in defining the differences between the crime and investigation as far as such investigation is concerned.

3. Controlled delivery. In the context of the rule of law, the lawful act of controlled delivery complies more with the values pursued by the criminal procedure, if comparing with that of allured investigation, from the perspective of crime punishment and procedural fairness. But the investigators who are influenced by the detection-centred doctrine have worries in their mind about the disadvantageous aspects of the controlled delivery, which will consequently bind their hands and feet in the criminal investigation.

\section{Countermeasures and Suggestions in Increasing the Effectiveness of Tracking Down the Upper-reaches and Lower-reaches Suspects in Drug-related Cases}

Generally speaking, the investigation organ should use reasonable investigation measures within a reasonable period of investigation in order to maximize the realization of the detection work. Here are some thoughts for further improvement in the detection of drug-related crimes:

\section{Dig Deeply and Expand the Lines and Establish a Drug-Control Information System in Shaanxi Province}

1. As far as the problems on the clues involving the upper-reaches and lower-reaches stated by the arrestees lacking of "directivity" and "accessibility" are concerned, we think, on the one hand, the investigators can improve their skills in interrogating the arrestees; on the other hand, they should attach importance to the collection of indirect evidence involving the arrestees in order to find some traces of the "upper-reaches and lower-reaches suspects". The indirect evidence plays corroborative 
role in judging the authenticity of criminal suspects' confessions and statements. Nevertheless, the investigation organ often ignores collection of such indirect evidence, which cast doubts on the authenticity of the confessions and statements made by the arrested suspects.

2. As for the problem of single crime clues on the upper-reaches and lower-reaches suspects in the mode of proactive investigation in drug-related cases, we argue to consider use for reference foreign best practices. For example, through the comparative study, we found that the well-established drug-control information system in the USA played a significant role when tracking down the upper-reaches and lower-reaches suspects in drug-related cases. Therefore, it is worthy discussing if a "Four (agencies) in One (platform)" drug-control information system could be established in Shaanxi Province. "Four (agencies)" means the staff and departments of secret and technical investigation, national security or headquarters and Provincial narcotics investigation in Shaanxi, who should implement the mechanisms of drug-related information sharing and immediate linkage in order to solve the problem of single source clue.

\section{Changing the Thoughts and Methods and Being Resilient in Tracking down the Crimes}

Since the upper-reaches in other provinces have certain connections with the drug dealers or addicts in Shaanxi Province rather than being isolated from each other, it is advisable for the investigators to remove their thought of local protectionism. In our view, the breakthrough point should focus on the "lower-reaches", particularly the drug addicts, as the latter are relatively weak against the public security organ from the antagonistic perspective and are easily to be discovered and subject to the surveillance. The investigation organ can trace the upper-reaches suspects in the drug-related crimes through the interrogation or secret follow-up tracing of the lower-reaches.

\section{Comprehensive Use of the Investigation Methods in Tracking down the Upper-Reaches and Lower-Reaches}

1. Making the information and materials of technical investigation lawful in the criminal procedure. There is a special section regarding technical investigation in the CPL (2012) which has defined the legality of such investigation used in important drug-related cases in China; however, the provisions are very rough and not so detailed, making it difficult in putting into effect. Therefore, when applying the technical investigation under the existing conditions, the narcotics squad and the technical investigation team are two relatively independent departments, who must cooperate with each other closely in order to avoid unnecessary delays with regard to the timing of detecting the drug-related crimes. Finally, as far as the transformation of evidence, the narcotics police transformed the information obtained through technical investigation into written materials so as to use these as evidence in the criminal procedure in past practice, which makes the legality and objectivity of technical investigation being challenged. As a result, it may be more scientific and reasonable if the technical investigation department can directly present the information and materials obtained as evidence.

2. Making good use of secret investigation. In our survey, some of the narcotics police officers in Shaanxi Province thought that secret investigation can easily tough the "high-voltage wire" of joint crime when tracking down the upper and lower-reaches suspects in drug-related cases, which needs further studies in the future. Moreover, it is advised to set up various secret investigators or informants to serve for tracking down the upper-reaches and lower-reaches in drug-related crimes. Finally, it is necessary to consider secret investigators or informants in places of drug source, if the timing is right and the conditions are available, in order to solve the disadvantageous situation in tracking down the upper-reaches.

3. Making use of the controlled delivery method. Controlled delivery has been affirmed by the United Nations Convention against Illicit Traffic in Narcotic Drugs and Psychotropic Substances, and has been adopted by the Narcotic Agencies in many countries. Drug trafficking often takes the form of separating the goods (drugs) and the real drug traffickers. The purpose of controlled delivery is to find out the conjunct point, to make a follow-up trace and even to extend the range of controlled delivery, which will be of great benefit to improve the ability of tracking down the trans-regional drug transactions between the upper-reaches and lower-reaches in Shaanxi Province. 
Along with the formation of the crime pattern of "delivering the drugs from the south to the north" coming into being, the narcotics departments in Shaanxi should have closer cooperation with those counterparts in the source regions of drugs such as Sichuan, Yunnan and Guangdong. On the basis of good police cooperation, the authorities in Shaanxi Province should take advantage of controlled delivery to put the upper-reaches and lower-reaches suspects into the scope of investigation, to master the rules of the drug trafficking chains, and to choose the best timing to obtain the conclusive evidence for tracking down the upper and lower-reaches suspects.

\section{Conclusion}

Based on the discussions above, it is necessary for China to improve the detective measures in striking down drug-related crimes. Given the limitation of the research sample, however, our study may not provide an overall picture regarding the reasons of the investigation organ's failure in detecting more suspects that they should do in China, including those upper-reaches and lower-reaches. This requires more and similar works to be done by us and other colleagues in the future. Nevertheless, we are confident that the effectiveness of detecting the drug-related cases will be improved if the investigators can change their stereotyped mind and make use of more scientific and technical skills, if supported by a sound drug-related information network.

\section{Acknowledgement}

This research was financially supported by the Human and Social Sciences Project of Shaanxi Department of Education "Criminal misjudged case and the mechanism of responsibility".(2017) Project Grant No. [17JK0526]

\section{References}

[1] Li, Dong (2014). "On Investigation of Optimization of Drug-related Crime Cases". Journal of Guangxi Police College (Guangxi Jingguan Gaodeng Zhuanke Xuexiao Xuebao) 3:1-5.

[2] Lu, Jia (2015). "Investigation Countermeasures for the Cases of Drug-related Crime in the Context of the New Criminal Procedure Law". Journal of Hubei University of Police (Hubei Jingguan Xueyuan Xuebao) 6:14-16.

[3] Hong, Yi (2013). "On the Characteristics of Drug Crimes and Allured Investigation", Legal System and Society (Fazhi yu Shehui) 132-134.

[4] Wang, Jiapeng (2012). “On the Legal Regulation of Temptation Investigation in Drug Crimes from the Perspective of the New Criminal Procedure Law". Legal System and Society (Fazhi yu Shehui) 289-290. 